Aim of the study: The present study aims to discuss the value and the effect of resection of suprasellar meningioma through the interhemispheric approach.

Material and methods: Twenty-nine cases of patients with suprasellar meningioma diagnosed through enhanced magnetic resonance imaging (MRI) scans and postoperative histopathological examination underwent resection of tumours (the largest diameter ranged from $3 \mathrm{~cm}$ to $6 \mathrm{~cm}$ ) by the microsurgical technique of a small bone window (about $4 \mathrm{~cm} \times 5 \mathrm{~cm}$ ) through the interhemispheric approach. Results: Among all cases, 25 (86\%) (Simpson I, II) were of total resection of tumours and 4 were of subtotal resection of tumours. 19 (65\%) were of improvement of vision and visual field, $2(7 \%)$ were of postoperative diabetes insipidus, and 1 (3\%) was of electrolyte imbalance. No operative death occurred.

Conclusions: The small bone window interhemispheric approach can be used to expose tumours, lightly stretch brain tissues, reduce the incidence of complications, and improve the total resection rate of tumours of patients with sellae meningiomas growing forward, upward, and into the sella.

Key words: suprasellar meningioma, small bone window, interhemispheric approach, complete resection.

Contemp Oncol (Pozn) 2013; 17 (6): 525-529 DOI: $10.5114 /$ wo.2013.38913

\section{Twenty-nine cases of resection of suprasellar meningioma through small bone window: an interhemispheric approach}

\author{
Zhao-Feng Lu, Xiao-bing Cheng, Yong-gang Zhao, Bao-zhong Shi
}

The First Affiliated Hospital of Henan Science and Technology University, China

\section{Introduction}

Suprasellar meningioma originates in the meningioma of the tuberculum sella, sellar diaphragm, anterior clinoid process, and sphenoidal planum, accounting for about $5 \%$ to $10 \%$ of intracranial meningiomas [1]. The tumour is located in the midline at the base of the skull and originates in the sella [2]. Its clinical manifestations are mainly monocular or binocular hypopsia and bitemporal hemianopsia, optic atrophy without papilloedema, and smell and mental disorders. However, some patients exhibit endocrine disorders [3]. Microsurgery resection should be preferred over therapy. Stirling and Edin described tuberculum sellae meningioma for the first time in 1897 [4]. This type of meningioma was resected initially by Cushing and Eisenhardt in 1916 [5]. Suprasellar meningioma originates in the meningioma of the sellar tuberculum, sellar diaphragma, anterior clinoid process, and sphenoidal planum [6], which was posited by Cushing in 1938 and categorised into early stage, prior stage of symptom, early stage of symptom, and operative effect stage [7]. Although there are different classifications of anatomical parts, selecting the surgical approach according to strict anatomical parts is more important because of the similar clinical syndromes caused by larger meningioma [8]. Several studies show that the total tumour resection rate has significantly increased, and the mortality rate has significantly decreased [9]. However, conflict remains regarding the relationship between the postoperative neurologic function, especially recovery of vision and view, and surgery [10]. Zevgaridis believed that the boundary of tumours and brain tissues is clear and that patients without preoperative severe loss of vision $(<0.2)$ would have excellent recovery [11]. Surgical approach and surgical skills are crucial to the resection of tumours and the protection of neurological function. Twenty-nine cases of microsurgery resection of suprasellar meningioma through the interhemispheric approach performed from January 2005 to December 2009 exhibited excellent curative effects.

\section{Material and methods}

General data

Twenty-nine patients diagnosed by pathological examination from January 2005 to December 2009 were retrospectively analysed. Among the cases, 10 were of males and 19 were of females aged 25 to 69 years old, with an average age of 47 . This study was conducted in accordance with the Declaration of Helsinki and with approval from the Ethics Committee of the First Affiliated Hospital of Henan Science and Technology University. Written informed consent was obtained from all participants. 


\section{Clinical manifestations}

Among the cases, all with different degrees and symptoms of headache and dizziness, 25 were of starting visual impairments, 2 of starting symptoms of headache, 1 of mental abnormality, 1 of infertility, and 1 of higher than normal prolactinaemia, as indicated by an endocrinological check-up.

\section{Imaging examination}

All patients underwent a preoperative magnetic resonance image scan (Figs. 1A, 1B, and 1C) and enhanced examination (Figs. 1D and 1E). Among the patients, 20 had tumours $3 \mathrm{~cm}$ to $5 \mathrm{~cm}$ in diameter and 9 had tumours equal to or larger than $5 \mathrm{~cm}$ in diameter. Moreover, 3 patients had violating cavernous sinus, 16 patients with internal carotid wrapped, 2 patients with growth in sella, 1 with growth in sphenoid sinus, and 2 patients with calcification in the tumour.

\section{Surgical treatment}

All patients underwent micro-neurosurgical resection through the small bone window interhemispheric approach under general anaesthesia. All 29 patients were confirmed to have meningioma through pathological examination, in which 14 cases were of the endothelial type, 7 cases of fibrous type, 6 cases of psammomatous type, and 2 cases of haemangioma type.

Flap and bone window: All patients were in the supine position, with head in the midline, in $20^{\circ}$ supine angle after being fixed by Mayfield three pin skull clamps. The head of the bed was lifted up by about $10^{\circ}$ to $15^{\circ}$. A curved incision about $10 \mathrm{~cm}$ to $15 \mathrm{~cm}$ was made inside the hairline of the circular crown turned over and overhung to the front. The periosteum was rectangularly cut off and turned over to the supraorbital margin. Pneumatic drill and milling cutters forming a rectangle crossed the midline, with a free bone flap of about $4 \mathrm{~cm}$ to $5 \mathrm{~cm}$ and the periphery not more than the bilateral supraorbital notch. The opening frontal sinus was blocked with bone wax and the mucosa was dissected. A pneumatic drill was used to grind crista bone.

Incision of the dura mater and treatment of the superior sagittal sinus: The dura mater was transversely cut off near the superior sagittal sinus, and the cerebral flax was cut off after clipping the sinus with vessel forceps. Haemostasis was performed with double ligation, and the bridging veins of the frontal lobe were retained furthest. The dura mater was stretched in the front and back directions. A self-retaining retractor (AESCULAP INTERNATIONAL GMBH, Germany) and surgical microscope (Leica M525 $\mathrm{OH} 4$, Germany) were used. While gently separating along the longitudinal direction, the cerebrospinal fluid was released. The self-retaining retractor was adjusted up to the exposed tumour, complex of tuberculum sella, optic chiasma, end plate, and anterior communicating artery. The dura mater was tightly sutured after tumour resection. The skull was fixed with a skull fixation system and then drainage was subcutaneously placed.
Exposure and resection of the tumour: The frontal lobe was gently separated by the self-retaining retractor. The tumour grew upward. It was red and tough with rich blood supply. It was closely related to the complex of the tuberculum sella, optic chiasma, lamina terminalis, and anterior communicating artery. The tumour was resected en bloc. The blood supply of the basal parts of the tumour was cut off. Intraoperative tumour tissues adhering to the important structures were carefully separated; the hypothalamus, perforating branch vessels, and pituitary stalk were carefully protected. The dura mater at the adherence point was fully fulgurated after tumour resection.

\section{Results}

\section{Operation effect}

All cases underwent magnetic resonance imaging (MRI) scan (enhanced). Among the cases, 25 cases (86\%) (Simpson I, II) had total resection of the tumour (Fig. 1F-I) and 4 cases (14\%) had subtotal resection. In the postoperative follow-up of 3 months to 13 months, 2 cases (7\%) of relapse were confirmed by MRI after about one year, without obviously aggravated symptoms. These cases were given further treatment using the $\boldsymbol{\gamma}$-Knife after their transfer to the Department of Radiotherapy.

\section{Vision and visual field}

Postoperative vision increase to 0.1 after 3 months was considered an improved condition. Among the patients, 19 $(66 \%)$ improved one month after the operation $(\geq 0.1)$, and 10 cases (35\%) did not exhibit obvious changes compared with their vision before the operation. None of the patients experienced visual deterioration in this group. Four cases (14\%) significantly improved 7 days after the operation (their vision improved by more than 0.3). Among 29 patients with view field defects, 20 cases (69\%) improved, and 9 cases (31\%) did not exhibit changes in the postoperative detection of vision field after one month. Ten cases (35\%) significantly improved 3 days after the operation.

\section{Postoperative complications}

Two cases of postoperative diabetes insipidus were cured after best support care for 3 weeks through the application of pituitrin and pituitary tannate. One case had postoperative mental symptoms manifesting euphoria and irritability but improved after antipsychotic treatment for 2 weeks. One case of postoperative hemiplegia for 3 weeks with grade 4 muscle strength in the hemiplegic side improved after rehabilitative and symptomatic treatment. No deaths occurred.

\section{Discussion}

Among the 29 cases, 16 (55\%) cases of the basilar part originated in the tuberculum sella and sellar diaphragm, and 3 (10\%) cases approached the sphenoidal planum simultaneously. However, in all cases the main part of the tumour grew upward. All cases had a visual disorder manifested by progressive vision loss, and visual field defect, especially bitemporally. Twenty-five (86\%) 

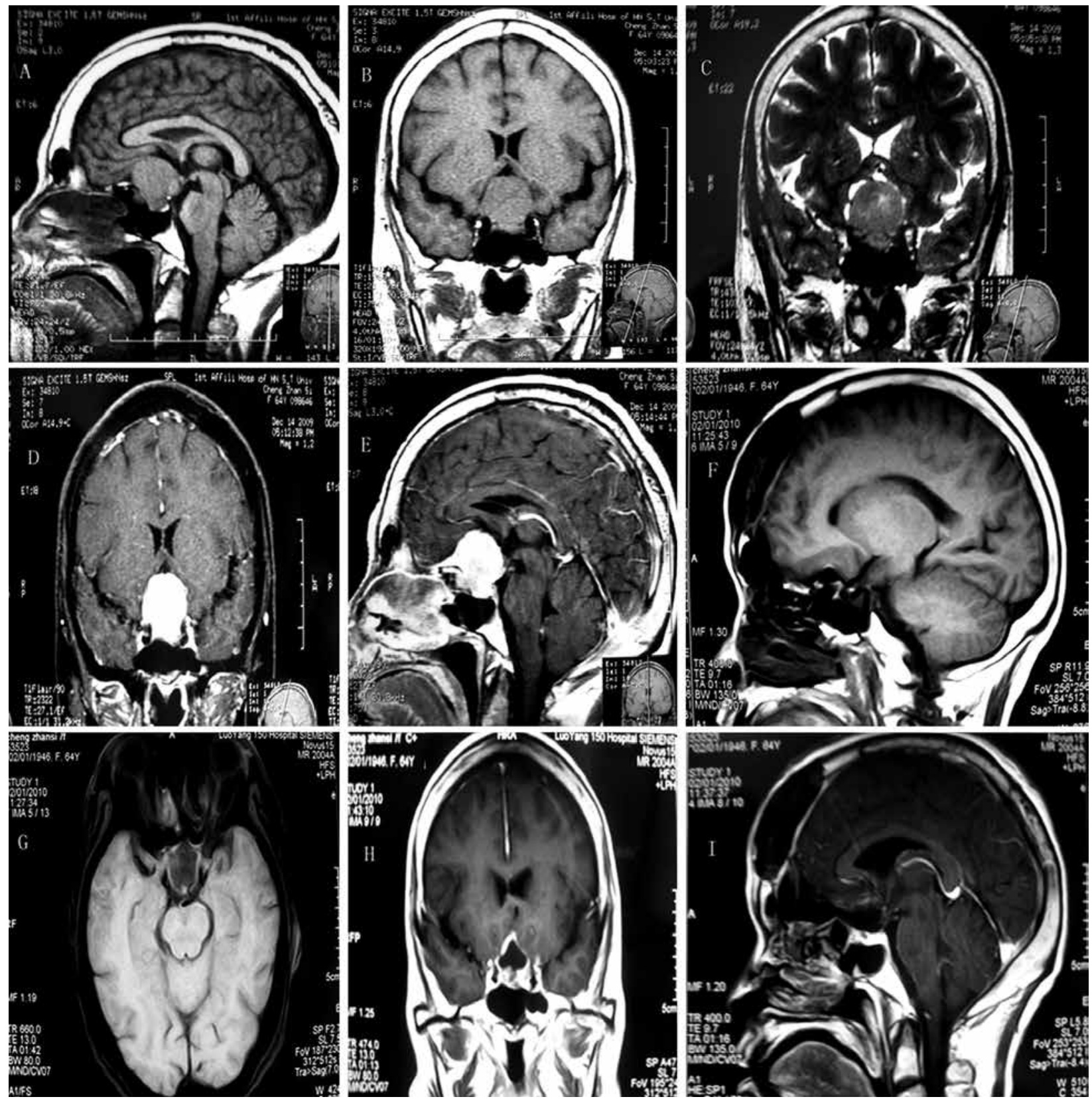

Fig. 1. Pre- and postoperative MRI of a female patient (64 years old) with suprasellar meningiomas. A) The preoperative MRI sagittal $\mathrm{T}_{1}$-weighted image showed the tumour located in the suprasellar region. B) The preoperative MRI coronal $\mathrm{T}_{1}$-weighted image showed the tumour located in the suprasellar region with optic chiasm compression. C) The preoperative MRI coronal $\mathrm{T}_{2}$-weighted image showed the tumour located in the suprasellar region with optic chiasm compression. D) The preoperative enhanced MRI coronal image (Magnevist) showed the tumour located in the suprasellar region with dural tail. E) The preoperative enhanced MRI sagittal image (Magnevist) showed the tumour located in the suprasellar region with dural tail. F) The postoperative 1 month MRI sagittal $\mathrm{T}_{1}$-weighted image demonstrated total removal of the tumour. G) The postoperative 1 month MRI transverse $\mathrm{T}_{1}$-weighted image demonstrated total removal of the tumour. $\mathrm{H}$ ) The postoperative 1 month enhanced MRI coronal image (Magnevist) demonstrated total removal of the tumour. I) The postoperative 1 month enhanced MRI sagittal image (Magnevist) demonstrated total removal of the tumour

cases were of abnormal vision as the first clinic symptoms, with varying degrees of headache and dizziness, symptoms in 2 cases started with headache, 1 of mental abnormality misdiagnosed as mental diseases and given surgical treatment after confirmation by MRI, and 1 case of infertility given surgical treatment after symptomatic treatment because of an abnormal endocrine check-up result and confirmed meningioma by pathological examination.
With the advances in anatomy of the skull base along with the improvement of surgical approaches and microneurosurgical techniques, the total resection rate of suprasellar meningioma and its prognosis have greatly improved [12]. Commonly used operation approaches include bilateral frontal, single frontal, and pterional approach. Resection of suprasellar meningioma through the endoscope-assisted supraorbital keyhole has also been conducted [13]. The single frontal approach exposes the su- 
prasellar meningioma well, but only for smaller tumours; it easily damages the frontal lobe and olfactory nerve. The optic nerve and areas below the optic chiasma are difficult to fully expose through the pterional approach [14]. There exists increased risk of diabetes insipidus and cerebrospinal fluid leakage through the bilateral frontal interhemispheric approach. Operation flexibility is reduced because of the limited visual field through the supraorbital keyhole approach [15]. There is less application of the forehead interhemispheric approach because of brain swelling, neurological function disorders, and other complications caused by intraoperative damage of the frontal lobe and bridging veins [16]. However, with the improvement of micro-operative technique and equipment, the forehead interhemispheric approach has become safe and effective [17]. The operation effect and prognosis of the 29 cases were improved further by the operation through a small bone window by the interhemispheric approach. The main operation points were as follows. 1) Small bone window. The size of the rectangle bone window across the midline was $4 \mathrm{~cm}$ to $5 \mathrm{~cm}$, not more than that of the bilateral supraorbital notch. 2) Low position. The incision was as close as possible to the anterior skull base. The open frontal sinus was washed with hydrogen peroxide and gentamycin saline and then blocked by a muscle flap. 3) Reduced stretch of the frontal lobe. Pay attention to protection of frontal lobe bridging veins and olfactory nerve along with a short distance to the tumour. 4) Clear exposure and wider surgical field of view. The upward sella, interior sella, and field of view beside the sella up to the interpeduncular fossa and the veutro of pons were fully exposed. While resecting tumours, the hypothalamus, pituitary stalk, and important perforating branches were protected fully under euthyphoria. Serious complications caused by hypothalamus injury and pituitary stalk injury were reduced. 5) Convenient operation. This method was convenient with the use of a surgical microscope and operation and in rebuilding the skull base postoperatively. Postoperative cerebrospinal leak and intracranial infection were further reduced. The first and second gap were the major operation space. The boundary of the tumour was clearly separated, then the tumour was resected en bloc. The tumour base could be completely resected within enough operation space. The dura mater attached to the tumour should be dealt with last if possible. The intraoperative bilateral optic nerves, optic chiasma, anterior communicating artery and anterior artery $A_{1} A_{2}$ segments, and posterior communicating artery were exposed as much as possible. The pituitary stalk and perforating arteries were protected fully. In our study, all 25 cases were fully exposed to protect the important structures during the total resection of tumours, because of large tumours and tight adhesion to the optic nerve and internal carotid.

Galal et al. studied 21 cases of suprasellar meningioma and found that all patients had disorders of vision and visual field defect [18], similar to the results in our study. Among the 17 cases of total resection of tumours and 4 cases of subtotal resection of tumours with follow-up for 28 months on average, 12 cases had improved vision (60\%), and 8 cases had no improvement (40\%). No worse cases had been recorded. The visual symptom schedule, tumour size, and relationship with peripheral vessels are important factors that influence the recovery of vision. Among the 29 cases, 19 had obviously improved vision (65\%) and 10 had no improvement (35\%). Four cases of patients significantly improved 7 days after the operation, with clearly improved vision (20/29) postoperatively. Therefore, the resection of tumours strictly along the arachnoid clearance is the most effective strategy to protect vessels and nerves.

Total resection of suprasellar meningioma has a good prognosis. Studies showed that the total resection rate depends on the relationship between the tumour and the important structures around it, such as the hypothalamus, blood vessels, optic nerves, and pituitary stalk [19]. The character of the tumour also influences the total resection rate [20]. Intraoperative protection surrounding important structures is the key to best prevent complications during total resection of tumours [21]. Among all the cases, 2 were of postoperative diabetes insipidus, 1 of postoperative mental symptoms, and 1 of postoperative hemiplegia, with grade 4 muscle strength in the hemiplegic side; all cases were caused by tough tumours and close connection with the pituitary stalk. The excessive stretch of the frontal lobe could lead to pituitary stalk symptoms. Three cases of subcutaneous effusion were caused by the relatively loose suture of the dura mater. The size of the tumour makes little difference to the incidence of complications [22].

The small bone window interhemispheric approach is a safe and convenient treatment of suprasellar meningioma with a high total resection rate. It could be improved further with endoscopy to target tumours specifically located in the parasellar region.

The authors declare no conflict of interests.

\section{References}

1. Suri A, Narang KS, Sharma BS, Mahapatra AK. Visual outcome after surgery in patients with suprasellar tumors and preoperative blindness. J Neurosurg 2008; 108: 19-25.

2. Dehdashti AR, Ganna A, Witterick I, Gentili F. Expanded endoscopic endonasal approach for anterior cranial base and suprasellar lesions: indications and limitations. Neurosurgery 2009; 64: 677-87.

3. Ceylan S, Koc K, Anik I. Extended endoscopic approaches for midline skull-base lesions. Neurosurg Rev 2009; 32: 309-19.

4. Chowdhury FH, Haque MR, Goel AH, Kawsar KA. Endoscopic endonasal extended transsphenoidal removal of tuberculum sellae meningioma (TSM): an experience of six cases. Br J Neurosurg 2012; 26: 692-9.

5. Chamoun R, Couldwell WT. Practical and technical aspects of trans-sphenoidal surgery. J Neurosurg Sci 2011; 55: 265-75.

6. Liu JK, Christiano LD, Patel SK, Tubbs RS, Eloy JA. Surgical nuances for removal of tuberculum sellae meningiomas with optic canal involvement using the endoscopic endonasal extended transsphenoidal transplanum transtuberculum approach. Neurosurg Focus 2011; 30: E2.

7. Zada G, Du R, Laws ER Jr. Defining the "edge of the envelope": patient selection in treating complex sellar-based neoplasms via transsphenoidal versus open craniotomy. Neurosurg 2011; 114: 286-300.

8. Dehdashti AR, Ganna A, Witterick I, Gentili F. Expanded endoscopic endonasal approach for anterior cranial base and suprasellar lesions: indications and limitations. Neurosurgery 2009; 64: 677-87. 
9. Fatemi N, Dusick JR, de Paiva Neto MA, Kelly DF. The endonasal microscopic approach for pituitary adenomas and other parasellar tumors: a 10-year experience. Neurosurgery 2008; 63: 244-56.

10. Cappabianca P, Cavallo LM, Esposito I, Barakat M, Esposito F. Bone removal with a new ultrasonic bone curette during endoscopic endonasal approach to the sellar-suprasellar area: technical note. Neurosurgery 2010; 66: E118.

11. Sekhar LN, Ramanathan D, Ferreira M. Postoperative visual outcome of suprasellar meningiomas. World Neurosurg 2011; 75: 219-21.

12. Selhorst JB, Chen Y. The optic nerve. Semin Neurol 2009; 29: 29-35.

13. Kaptain GJ, Vincent DA, Sheehan JP, Laws ER Jr. Transsphenoidal approaches for the extracapsular resection of midline suprasellar and anterior cranial base lesions. Neurosurgery 2008; 62: 1264-71.

14. Schwartz TH, Anand VK. The endoscopic endonasal transsphenoidal approach to the suprasellar cistern. Clin Neurosurg 2007; 54 226-35.

15. Maiuri F, Cappabianca P, laconetta G, Esposito F, Messina A. Simultaneous presentation of meningiomas with other intracranial tumours. Br J Neurosurg 2005; 19: 368-75.

16. Joseph V, Chacko AG. Suprabrow minicraniotomy for suprasellar tumours. Br J Neurosurg 2005; 19: 33-7.

17. Dusick JR, Esposito F, Kelly DF, Cohan P, DeSalles A, Becker DP, Martin NA. The extended direct endonasal transsphenoidal approach for nonadenomatous suprasellar tumors. I Neurosurg 2005; 102: 832-41.

18. Galal A, Faisal A, Al-Werdany M, El Shehaby A, Lotfy T, Moharram H. Determinants of postoperative visual recovery in suprasellar meningiomas. Acta Neurochir (Wien) 2010; 152: 69-77.

19. Presutti L, Trani M, Alicandri-Ciufelli M, Marchioni D. Exclusive endoscopic removal of a planum sphenoidale meningioma: a case report. Minim Invasive Neurosurg 2008; 51: 51-3.

20. Jalali R, Srinivas C, Nadkarni TD, Rajasekharan P. Suprasellar haemangiopericytoma - challenges in diagnosis and treatment. Acta Neurochir (Wien) 2008; 150: 67-71.

21. Kitano M, Taneda M, Nakao Y. Postoperative improvement in visual function in patients with tuberculum sellae meningiomas: results of the extended transsphenoidal and transcranial approaches. J Neurosurg 2007; 107: 337-46.

22. Laufer I, Anand VK, Schwartz TH. Endoscopic, endonasal extended transsphenoidal, transplanum transtuberculum approach for resection of suprasellar lesions. J Neurosurg 2007; 106: 400-6.

\section{Address for correspondence}

\section{Prof. Zhao-Feng Lu}

The First Affiliated Hospital

of Henan Science and Technology University

No. 24 Jinghua Road Jianxi District

471003 Luoyang, China

e-mail: zfbzcn@yeah.net

Submitted: 4.07.2012

Accepted: $\quad 16.10 .2013$ 